\title{
Determinants of the behavioral effects of opioids and their antagonists: contributions of the Laboratory of Psychobiology
}

Received: 1 April 2002 / Accepted: 13 July 2002 / Published online: 29 August 2002

(C) Springer-Verlag 2002

\begin{abstract}
Rationale: The behavioral pharmacology of opioids has been influenced significantly by the research and writings of Drs. Peter B. Dews, Roger T. Kelleher, and William H. Morse, their colleagues, and their students. Objective: Their conceptual and methodological approach to the topic is reviewed briefly, and three areas of research are described to provide an empirical perspective. Results: The objective of determining the general effects of opioids on behavior is described; the effects of opioids on schedule-controlled behavior and punished behavior are described and compared to nonopioids. The differential effects of opioid antagonists on responding reinforced by different stimuli are also presented. Conclusion: The conceptual and methodological approach taken by the group, as well as their discoveries in the behavioral pharmacology of opioids, will continue to exert a positive influence on the field.
\end{abstract}

Keywords Morphine - Analgesia · Opioid · Opioid antagonists $\cdot$ Operant conditioning

\section{Introduction}

The major members of the Laboratory of Psychobiology, Drs. Peter B. Dews, Roger T. Kelleher, and William H. Morse, had strong interests in opioids throughout their careers. Certainly of the three, Morse was the most interested in these drugs, and he contributed a number of seminal experimental papers that will be discussed in more detail later in this contribution.

\footnotetext{
J.H. Woods ( $\bullet)$

Department of Pharmacology, 1301 MSRB III,

University of Michigan Medical School, Ann Arbor,

MI 48109-0632, USA

e-mail: jhwoods@umich.edu

Fax: +1-734-7647118

C.P. France

Department of Pharmacology,

University of Texas Health Science Center, 7703 Floyd Curl Drive, San Antonio, TX 78229-3900, USA
}

Before describing their contributions to opioid behavioral pharmacology, it should be noted that they made a remarkable place for themselves among all individuals interested in drugs and behavior. They saw the question of how drugs affect behavior as fundamental. One can seek advice from chemical or biochemical colleagues, but they cannot answer the question of how drugs change behavior in general ways. Likewise, one can pay close attention to those who apply drugs in medical settings, for they make unique contributions to the description of how drugs change behavior in those settings. The clinician seldom has the luxury of looking at a drug's effects in a wide variety of behavioral circumstances. Thus, the task of describing the behavioral substrate of drug action falls directly upon the behavioral pharmacologist, and the colleagues of the Laboratory of Psychobiology believe that drug effects on conditioned behavior are necessary for understanding drug effects in vivo. Other contributions to the Festschrift will speak to other aspects of their contributions.

We think that Dews' 1974 paper entitled "What is Analgesia?" describes an intellectual context for studying opioids that is as vital today as when written, and that a discussion of this paper would illustrate the novelty and strength of the experimental disposition that was shared by all three investigators. What was Dews up to in raising the question of the nature of analgesia? At first glance, it might appear to be a curious question since virtually everyone would agree that morphine is particularly effective in dampening responsiveness to painful stimuli. The clinician's view of morphine, as a reliever of pain, is unquestioned; it remains the standard of reference!

When considered in a broader critical context, however, there was good reason to question the accuracy of labeling morphine an analgesic. Dews argued that, unlike acetylsalicylic acid, there was no strong evidence that morphine had a selective action on nocicepter excitation; moreover, the behavioral actions of morphine did not appear to be unique to situations involving painful stimuli. On the one hand, morphine had nearly identical effects on behavior that was maintained by either painful 
or non-painful stimuli. For example, the potency of morphine in decreasing responding maintained by food presentation or by electric shock termination was nearly identical. On the other hand, the effects of morphine were not the same on all behavior that was controlled by painful stimuli. For example, responding that was suppressed by the response contingent presentation of painful stimuli was not normalized by administration of morphine (see below). However, other drugs that typically were not considered to be analgesics (e.g. barbiturates) restored this suppressed responding to normal levels. Finally, Dews noted that the pain-relieving effects of morphine were remarkably similar under widely varying conditions of pain intensity. Thus, he argued that the behavioral pharmacology of morphine did not appear to be linked to pain or painful stimuli and, further, that there did not appear to be any unique aspect to the behavioral or general pharmacology of morphine that required the presence of pain. Was he being cantankerous or heretical? Neither - but stay with us.

What did Dews suggest that "analgesic" assays were measuring? He argued that assays and the manner in which they were used were contrived; those who were specifically interested in identifying morphine-like compounds excluded assays that failed to detect the effects of morphine and retained assays that were "sensitive" to the effects of morphine. A similar exclusionary approach to "method development" is evident throughout the history of psychopharmacology, including the selectivity and sensitivity of the forced swim test to various drugs that have antidepressant activity in humans (e.g. Porsolt et al. 1977; Reneric and Lucki 1998) and the selectivity and sensitivity of the Geller-Seifter assay to identify various drugs that have anti-anxiety effects in humans (e.g. Geller and Seifter 1960; Cook and Kelleher 1962; Barrett 1987). In both examples the value of the assay and its inclusion for future studies was determined based on its sensitivity to drugs that were pharmacological equivalents of a prototypes (imipramine and chlordiazepoxide, respectively) and its insensitivity to drugs that were not. The point of the argument regarding morphine and analgesia appears to have been not whether this type of "method development" was valuable - clearly it was. Nevertheless, the identification of assays that were behaviorally selective did not, for Dews, represent a solution to the more basic question of guiding research toward a general understanding of how morphine changed behavior in all situations.

So, if morphine does not affect all behavior that is under control of painful stimuli and if the effect of morphine is strikingly similar in conditions involving painful stimuli and those that do not, might it not be the case that morphine has a global effect on behavior, perhaps generated in different situations and by a variety of stimuli? Dews suggested that it could as easily be argued that morphine dampened distress as it could be argued that morphine was analgesic. He also suggested specific experimental questions that might be especially insightful with regard to whether morphine confers analgesic effects per se. For example, there are many situations in which behavior can be controlled by aversive or painful stimuli. Morphine had not been studied in many of these situations and in the cases where it had been studied it was not always clear that analgesia was the selective or dominant effect.

It is clear, reading today, that his intent was to stimulate rigorous studies on the topic. Did he think that the effects of morphine on distress would be similar to its effects under other conditions where acute pain was involved? Probably not. More likely he was trying to focus research questions on behavioral issues that would clarify why morphine is such a remarkable drug in the treatment of pain. In so doing he articulated the argument that scientists do themselves and their colleagues a disservice when they label drugs categorically; by so doing they dramatically increase the possibility that they will decrease the breadth and acuity of their investigations. In a different domain, some benzodiazepines have sedative and hypnotic effects; however, they have many other effects as well and, to the extent that calling them sedative/hypnotics limits the range of conditions over which they are studied, the label can be misleading.

Collectively, Dews argued cogently that morphine and morphine-like drugs had not been proved to attenuate selectively painful stimulation in either experimental subjects or in clinical situations. Moreover, he suggested instead that morphine had its major effect by relieving distress regardless of whether the distress arises from pain or other sources. His point, however, was not an attempt to divert researchers from studying the effects of morphine on pain or distress. Rather, it was a call to study all of the behavioral consequences of painful and non-painful stimuli and to study morphine (and other drugs) under an appropriately broad set of conditions that were neither restricted nor determined by preconceived notions regarding particular effects.

The effects of morphine depend on behavioral history; this must be kept in mind in order to gain insight into the nature of its effects. However, the study of morphine in this broader context is not sufficient; he argued that "the physiological and behavioral effects of morphine are too various to be subserved by a single principle" (p. 240). Thus, the simple study of its effects in contrived assays of analgesia in animals was not a substitute for a rigorous, balanced behavioral analysis of morphine's actions.

He remained unconvinced that even a better understanding of analgesia was the goal. He argued for "broad studies, unfettered by prejudice based on the names of supposed effects. We need to know the relevant characteristics of situations in which morphine, pentobarbital, and chlorpromazine are similar and of situations in which they differ. We need to develop an understanding of the relevant situational and behavioral factors that influence the behavioral effects of morphine, so that, eventually we can make some general statements about the effects (p 242)."

Thirty years of studies on the behavioral pharmacology of opioids has brought us a considerable distance in our 
Fig. 1 Representative cumulative records of performances under the multiple FR30, FI5 schedule after control and drug injections. Abscissa: time. Ordinate: cumulative number of responses. The FR and FI components are alternated; the components change after the presentation of food (short diagonal lines on the cumulative record) or after an elapsed time of 4 min during the FR component or after the end of the FI component (not shown). Note that each drug increased the level of responding during the FI component at the lower dose shown and decreased responding in both FI and FR components at the higher dose. In many instances responding was better sustained during FR components than during FI components. From McMillan and Morse (1967)

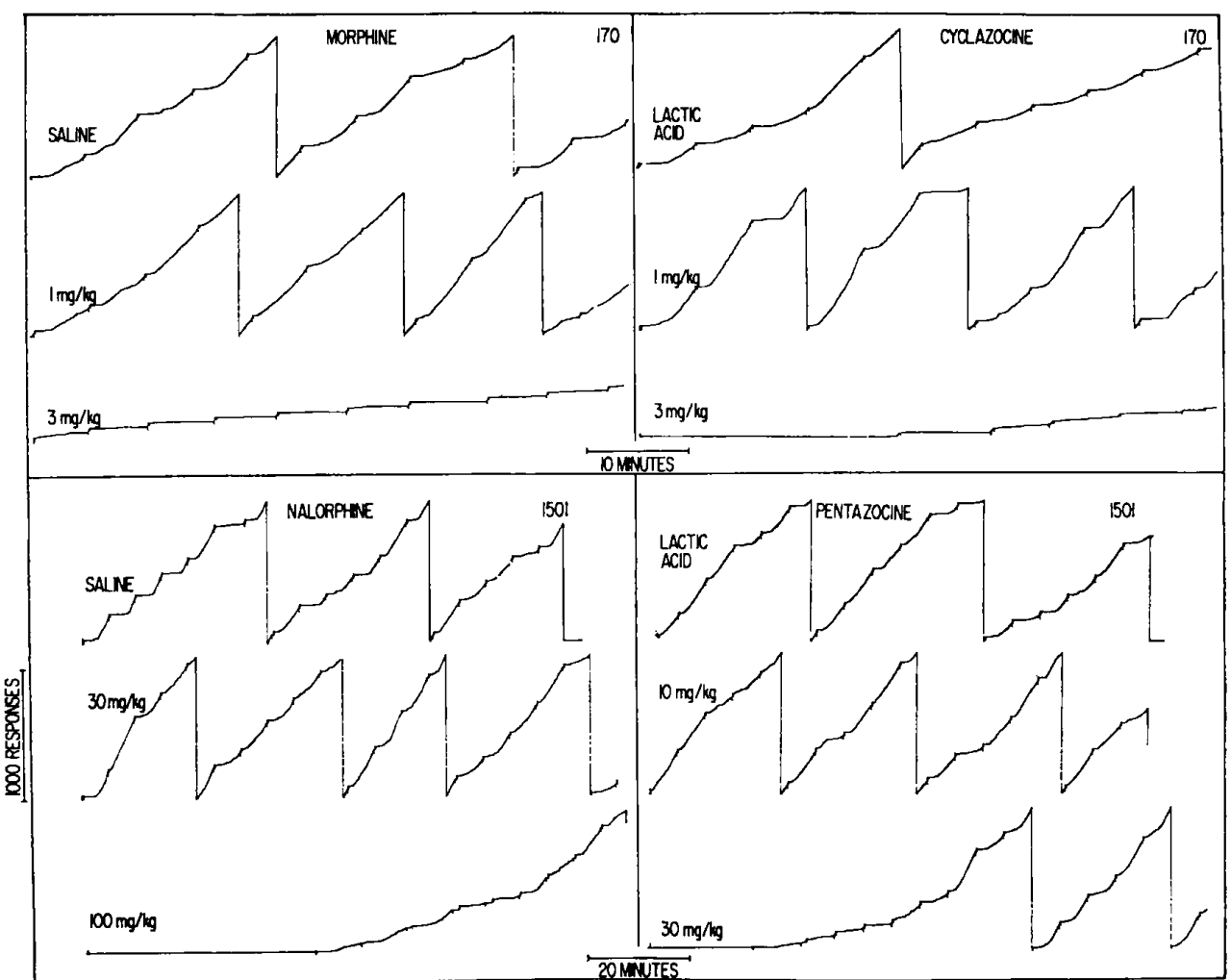

understanding of the behavioral pharmacology of morphine. We understand far more about the relative doses by which morphine exerts its various behavioral effects in a variety of organisms, including humans. It is reasonable to argue that we have not reached an improved understanding of the nature of its clinical essence - which was one of his objectives (and hopes!). Nevertheless, behavioral description of opioid effects has advanced the field and played an important role in the discovery of multiple opioid receptors, the effects of selective receptor antagonists, and insurmountable antagonists, and the discovery of agonists that function at restricted sets of receptors (e.g. peripheral). Thus, Dr. Dews' challenge to improve upon the behavioral account of morphine's clinical essence, in our opinion, is as relevant today as when it was written.

Whereas many different drugs could have been used to make the general argument against the labeling of drugs and for the global evaluation of their pharmacology, the selection of morphine appears to have been especially apropos because there are few drugs for which one might obtain so much agreement, among clinicians, scientists, and laypersons about the drug's (most common or important) effect. Nearly everyone would agree that morphine is an analgesic because it relieves pain. If this contribution has raised even the slightest doubt that you really understand the "analgesic" action of morphine, then one of Dews' major objectives will have been met. Behavioral analysis of drug effect was a major intellectual occupation of the members of the Laboratory. We decided to illustrate their approach and interests in opioids further by describing some selected experiments and the findings therefrom.

All of the contributions are characterized by the following: 1) outstanding experimental control of the behavior studied with very clear descriptions of the procedures utilized; 2) unusual attention to behavioral variation when rendered different by environmental circumstance including conditioning history of the subject; 3) careful consideration of dose-effect analysis of the drugs under study; and 4) inclusion of appropriate comparisons to drugs of conceptual and practical interest. In other words, the basics of the field!

The Laboratory, comprising three mentors and their students and staff, developed interesting approaches to the general issues of how opioids affect conditioned behavior, and we have divided these approaches into three topics: 1) effects of opioid agonists on schedule-controlled behavior; 2) effects of morphine on punished responding; and 3) effects of opioid antagonists upon behavior controlled by different reinforcers.

\section{Effects of opioid agonists and schedule-controlled responding}

Other contributors to this Festschrift have certainly indicated that schedule-controlled responding was a major focus of research during the formative period of the Laboratory of Psychobiology. Opioids were the subject of 
an early publication by D.E. McMillan, then a postdoctoral student, and Morse (McMillan and Morse 1967). Pigeons were the subjects of choice for the study since a number of investigators had used this species for studying other drugs. There had been a few studies of opioids on conditioned behavior reported in the literature at this time, but none was devoted specifically to a behavioral characterization of the effects of these drugs on schedule-controlled behavior. Perhaps, as suggested by the authors, there was a prevalent view that obtaining orderly dose-effect relations might be difficult due to the profound tolerance that was expected to develop from the repeated administration of opioids to the same subject. A moderately complex schedule of food-reinforced key pecking was conditioned in the pigeons with fixedinterval components alternating with small fixed-ratio components, each component associated with different colored key illumination. Four opioids were studied; morphine, nalorphine, cyclazocine, and pentazocine, each topically interesting at the time to a general pharmacological audience for a variety of reasons. Each compound was studied at several doses and the behavioral effects of the compounds were replicated within subjects. The opioids had reliable effects on responding: at small doses, each compound slightly increased responding in the fixed-interval component and, at larger doses, each suppressed both fixed-interval and fixed-ratio responding (Fig. 1). The potency order of these compounds in modifying schedule-controlled responding was similar to that shown in other assessments of behavioral activity as well as in assays of analgesia. Thus, the authors drew attention to the distinct possibility, born out by subsequent research, that the analgesic (antinociceptive) actions of opioids occurred at the same doses that affect behavior that is unrelated to reduction of pain or modulation of painful stimuli - a chord also played, as mentioned above, by Dews in other contexts.

When opioids were administered once weekly by McMillan and Morse, reliable effects of the same dose were obtained repeatedly. On the other hand, when opioids were administered more frequently their behavioral effects were diminished, indicating that tolerance could also be established, maintained and studied in pigeons. Thus, the resumed apprehension regarding tolerance induction was not born out; indeed, these studies formed the basis for a large number of studies that characterized both tolerance and dependence to opioids in a variety of species using the same type of general methodology. Indeed, a recent cross-reference of schedule-controlled responding and opioids yielded nearly a thousand citations.

\section{Effects of morphine-like agonists on punished responding}

Kelleher and Morse (1964) published a short review in which they raised a question regarding the manner by which drugs affected behavior that was strengthened and

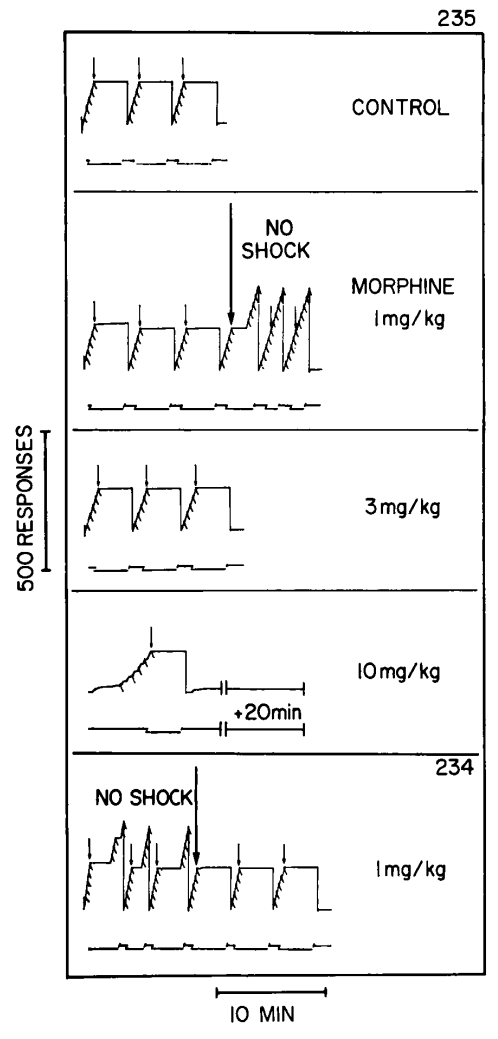

Fig. 2 Effect of morphine on behavior suppressed by punishment. The first four frames show performance of bird 235 on the fixedratio procedure in which non-punishment and punishment components alternated. Shocks were scheduled in punishment components except where indicated. The first record shows control performance. The start of the second record shows performance on the same procedure following the intramuscular injection of morphine $(1 \mathrm{mg} / \mathrm{kg}) ; 20 \mathrm{~min}$ in which no responses occurred have been omitted from the $10 \mathrm{mg} / \mathrm{kg}$ record. The lower frame shows the effect of morphine on the performance of bird 234. Scheduled shocks were omitted at the beginning of the record and then reinstated at the large arrow. Note that morphine did not prevent the almost immediate return of suppression by punishment with electric shocks. From Kelleher and Morse (1964)

maintained by the termination of aversive stimuli (escape behavior) and behavior that was suppressed by the presentation of aversive stimuli (punished behavior). Since we are restricting our comments to opioids, we will simply make their point that when behavior was suppressed by punishment, there were a variety of drugs that restored this behavior (pentobarbital and some drugs used at the time for the treatment of anxiety, e.g. meprobamate). Morphine failed to do so even when the behavior was only mildly suppressed by the conditions of punishment (Fig. 2). The authors also provided examples of situations in which a history of punishment continued to suppress behavior. For example, morphine could restore the suppressed behavior, but even in the presence of the morphine-induced restoration, the conditions of punishment could be reinstated and behavior would be suppressed (bottom frame, Fig. 2). On the other hand, pentobarbital readily restored responding under a broad 
range of conditions. At the time, the emphasis was on drugs that appeared to exert their effects on behavior in a manner that depended upon the contingency of reinforcement and little (or not at all) on the motivational state of the subject (e.g. fear and anxiety). To evaluate this hypothesis required that behaviors controlled by negative and positive reinforcers be made comparable in rate and pattern. To satisfy this requirement, behavior contingencies of reinforcement needed to be studied extensively; something that would try the patience of many. The rigorous and novel use of contingencies of reinforcement to formulate superior behavioral endpoints for drug study are as impressive today as they were when first published.

\section{Effects of opioid antagonists on responding maintained by different reinforcers}

During an especially productive period of the Laboratory, Kelleher and Morse wrote a series of influential papers in which they showed that amphetamine and chlorpromazine did not produce reinforcer-specific changes in behavior. They compared the effects of drugs on responding that were maintained by different reinforcers, particularly responding maintained by food presentation and responding maintained by escape from stimuli associated with occasional presentations of brief electric shock (e.g. Kelleher and Morse 1964). They found that the effects of drugs depended more upon the pattern of conditioned responding generated by the schedule rather than on the type of reinforcer. For example, morphine and U50488H, mu and kappa opioid receptor agonists, had similar effects (e.g. France and Morse 1989) on responding that was maintained by either of these reinforcers (lower panel, Fig. 3). However, the effects of opioid antagonists on scheduled-controlled behavior did not follow the precedent established by earlier studies on other classes of drugs.

Warren and Morse (1989) studied the effects of naltrexone on responding maintained by schedules of electric shock postponement, schedules involving escape from stimuli associated with electric shock presentation and schedules of electric shock presentation. Even at very large doses, naltrexone failed to suppress responding that was maintained under schedules utilizing shock, whereas these doses of naltrexone had marked rate-decreasing effects on responding which was maintained by the presentation of food. France and Morse (1989) compared fixed-ratio responding maintained by escape from stimuli associated with shock with fixed-ratio responding maintained by food presentation. Doses of naltrexone that eliminated responding maintained by food had relatively little effect on responding maintained by shock (upper panel, Fig. 3). Subsequently, it was shown that behavior maintained by a variety of different positive reinforcers, including some drug reinforcers, appears to be reduced by the same doses of naltrexone that decreased foodmaintained responding (e.g. Williams and Woods 1999). The characteristics of positive reinforcers that confer
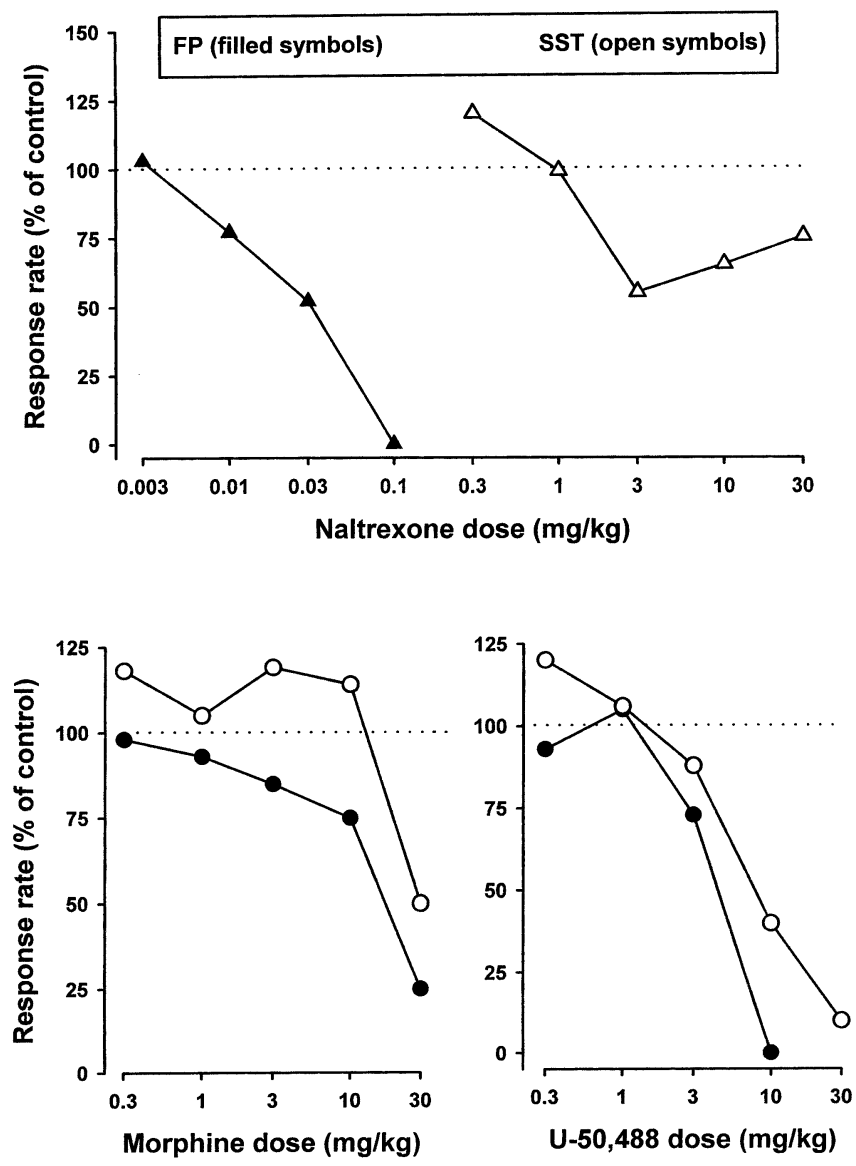

Fig. 3 Effects of naltrexone, morphine and U50,488H in squirre monkeys responding under fixed-ratio schedules of food presentation (FP, filled symbols) or positive reinforcement or stimulusshock termination (SST, open symbols), or negative reinforcement. Ordinates: average rates of responding, expressed as a percentage of the control rates. Abscissa: cumulative dose in $\mathrm{mg} / \mathrm{kg}$. Modified from Figs. 1 and 5 of France and Morse (1989)

susceptibility to behavioral actions of naltrexone have not been delineated, although it is clear that this susceptibility is not specifically linked to food reinfocers.

When naltrexone was administered repeatedly to the same subject, a marked supersensitivity develops (e.g. Spealman et al. 1981). Even very occasional (e.g. weekly) administration of an antagonist can generate large (e.g. 30-fold) increases in sensitivity (France and Morse 1989). The characteristics of compounds that share supersensitivity with naltrexone (i.e. cross-supersensitivity) have been studied. A variety of opioid antagonists (e.g. naloxone, nalmefene, diprenorphine, quadazocine, bremazocine, nalorphine, and MR 2266) share supersensitivity with naltrexone, whereas quaternary naltrexone (Warren and Morse 1985) and pentazocine (France and Morse 1989) do not. This effect (cross-supersensitivity) appears to be stereoselective in that naltrexone-sensitized subjects are also supersensitive to active isomers of opioid antagonists and not to the inactive (non-opioid) isomers (France and Morse 1989). Subjects sensitized to naltrexone are also supersensitive to lithium carbonate but not to 
kappa opioid agonists nor to a host of non-opioids (e.g. pentobarbital, phencyclidine).

Although the variables that contribute to the magnitude of supersensitivity have not been studied in detail, enhanced sensitivity to antagonists appears to remain very stable in non-human primates after repeated drug administration. It is remarkably persistent in the absence of intervention and it can still be observed 4 or more years after the establishment of supersensitivity (Kelleher and Goldberg 1979). Normal sensitivity to antagonists can be restored by conditioning the same response with an escape contingency (Warren and Morse 1989) or by acute administration of chlordiazepoxide (Warren and Morse 1985). More recently, others have suggested that GABAergic systems might play a role in the development of naltrexone supersensitivity since chlordiazepoxide restores it (Schindler et al. 1992) and that conditioning might also be an important component to the opioid antagonist induced supersensitivity (Schindler et al. 1990).

These behavioral actions of opioid antagonists remain a rich and interesting set of observations. It is possible that they may be related to the therapeutic effects of naltrexone in other contexts (e.g. alcohol dependence), although this possibility remains to be explored fully. Finally, it is ironic that supersensitivity in other areas of behavioral pharmacology (e.g. stimulants) is much less robust than what is unanimously observed with opioid antagonists, yet stimulant sensitization has received far more attention empirically and theoretically.

The members of the Laboratory of Psychobiology had a deep respect for the therapeutic effects of drugs, although this was not the explicit focus of their research. Rather, they studied the behavioral effects under wellcharacterized and highly controlled experimental conditions. The goals of this research did not typically include elucidation of mechanism in the sense of postulating specific receptors or systems that mediated drugs effects. Collectively, these pioneering investigators initiated, developed, and disseminated to the scientific community an approach to behavioral pharmacology that had the goal of understanding with considerable precision and great detail the behavioral actions of drugs. They fostered a rigorous, parametric approach that relied upon careful determinations of complete dose-effect relations, comparisons among drugs that varied in their behavioral actions, and comparisons among different experimental conditions, all with an unprecedented attention to the details of the conditions under which drugs were studied. The degree of rigor that was characteristic of behavioral studies in the Laboratory of Psychobiology spawned a variety of new ways of studying the behavioral effects of opioids. Unprejudiced investigation of the behavioral pharmacology of opioids without constraints of drug labels or procedural biases will probably continue to facilitate a much better understanding of and appreciation for the various components of action of morphine and other opioids that contribute to their analgesic and other behavioral effects.

Acknowledgements The authors would like to express their appreciation for the suggestions of the editors of this series. The research described herein was supported by USPHS Grant DA00254 and DA-05018. C.P.F. is the recipient of a Research Scientist Development Award, DA-00211.

\section{References}

Barrett JE (1987) Nonpharmacological factors determining the behavioral effects of drugs. In: Meltzer H. (ed) Psychopharmacology: a third generation of progress. Raven Press, New York, pp 1493-1502

Cook L, Kelleher RT (1962) Drug effects on the behavior of animals. Ann NY Acad Sci 96:315-335

Dews PB (1974) What is analgesia? In: Braude MC, Harris LS, May EL, Smith JP, Villarreal JE (eds) Narcotic antagonists (advances in biochemical psychopharmacology, vol 8). Raven Press, New York, pp 235-243

France CP, Morse WH (1989) Pharmacological characterization of supersensitivity to naltexone in squirrel monkeys. J Pharmacol Exp Ther 250:928-936

Geller I, Seifter J (1960) The effects of meprobamate, barbiturates, $d$-amphetamine, and promazine on experimentally induced conflict in the rat. Psychopharmacology 1:482-492

Kelleher RT, Goldberg SR (1979) Effects of naloxone on scheduled-controlled responding. In: Usdin E, Bunney WE, Kline NS (eds) Endorphins in mental health research. Oxford University Press, New York, pp 461-472

Kelleher RT, Morse WH (1964) Escape behavior and punished behavior. Fed Proc 23:808-816

McMillan DE, Morse WH (1967) Some effects of morphine and morphine antagonists on schedule-controlled responding. J Pharmacol Exp Ther 157:175-184

Porsolt RD, LePichon M, Jalfre M (1977) Depression: a new animal model sensitive to antidepressant treatments. Nature 266:730-732

Reneric J-P, Lucki I (1998) Antidepressant behavioral effects by dual inhibition of monoamine reuptake in the rat forced swimming test. Psychopharmacology 136:190-197

Schindler CW, Wu XZ, Su TP, Goldberg SR, Katz JL (1990) Enhanced sensitivity to the behavioral effects of naltrexone in rats. J Pharmacol Exp Ther 252:8-14

Schindler CW, Morley RJ, Goldberg SR (1992) Enhanced sensitivity to naltrexone is associated with an up-regulation in GABA receptor function. Life Sci 50:PL1-PL6

Spealman RD, Kelleher RT, Morse WH, Goldberg SR (1981) Supersensitivity to the behavioral effects of opiate antagonists. Psychopharmacol Bull 17:54-56

Warren PH, Morse WH (1985) Effects of quaternary naltrexone and chlordiazepoxide in squirrel monkeys with enhanced sensitivity to the behavioral effects of naltrexone. J Pharmacol Exp Ther 235:412-417

Warren PH, Morse WH (1989) Environmental determinants of enhanced sensitivity to the behavioral effects of naltrexone. J Pharmacol Exp Ther 248:546-551

Williams KL, Woods JH (1999) Naltrexone reduces ethanol- and/or water-reinforced responding in rhesus monkeys: effect depends upon ethanol concentration. Alcohol Exp Clin Res 23:14621467 